\title{
Microscopic lesions in the brain following head injury
}

\author{
D. R. OPPENHEIMER
}

From the Department of Neuropathology, Radcliffe Infirmary, Oxford

In cases of head injury with more than a few hours' survival, microscopic examination of the brain may reveal lesions which are not apparent on naked-eye inspection. In the first place, anoxic cell changes are frequently seen in cases where the injury has been complicated by respiratory troubles. Secondly, there are diffuse microscopic lesions attributable to accelerations within the brain substance at the time of injury. These may be in the form of multiple capillary haemorrhages-which to the naked eye are often indistinguishable from the normal vascular markings of a congested brain-or in the form of microscopic disruptions of nervous tissue, without haemorrhage. It is the purpose of this paper to show that the use of a simple silver impregnation enables one to pick out these tiny lesions with the scanning power of the microscope. It is suggested that a study of the sites, numbers and distribution of these lesions might throw useful light on the mechanisms of acceleration injuries to the brain.

\section{METHODS}

The brains from cases of head injury are examined after two to four weeks' fixation in $10 \%$ neutralized formalin. They are sliced, and the naked-eye lesionscontusions, lacerations, haemorrhages, softenings, internal tears, herniations, etc.-are noted. In most cases, blocks are taken for histology, the main points of interest being:

1. The presence or absence of 'anoxic' cell changes, and of areas of acute infarction which are not recognized by palpation or naked-eye inspection;

2. The presence or absence of diffuse capillary haemorrhages, indistinguishable by naked eye from normal vascular markings;

3. In cases with long survival, the distribution of tract degenerations and of the diffuse white matter lesions described by Strich (1956 and 1956a);

4. In cases with shorter survival, the presence or absence of foci of microglial reaction.

The first two of these investigations are readily carried out in conventional stains on paraffin sections. The third requires a combination of stains for myelin, fat, Marchi products, and neuroglia. The present paper is not directly concerned with these three lines of inquiry, but with the fourth. The material is drawn from 59 cases of head injury, with clinical features ranging from mild concus- sion to virtual decerebration, and survival times from 12 hours upwards. The cases constitute about a third of the number of brains from head injury cases examined in a period of six years. As the object of the inquiry was not primarily statistical, the selection of cases was not rigid or consistent. In general, those with gross haemorrhagic or destructive lesions were excluded, as well as those with survival periods of less than 12 hours.

The method is to examine frozen sections stained by a modification of the Weil and Davenport (1933) technique for microglia and oligodendroglia, which is as follows:

(1) Cut frozen sections at $20-25 \mu$; (2) Wash twice in distilled water; (3) Impregnate $10-20 \mathrm{sec}$ in ammoniacal silver bath made up as follows: to $2 \mathrm{ml}$. ammonia add $10 \%$ silver nitrate until solution is faintly opalescent (approx. $18 \mathrm{ml}$. silver nitrate). (4) Reduce in $10 \%$ formalin, keeping the section moving by blowing on surface of fluid. (5) Wash in distilled water. (6) Dehydrate to absolute alcohol and clear in carbol-xylol. Mount in Xam.

The background is stained light brown. Microglial cells and their processes are dense black, and are still partially stained in the lipid phagocyte stage. Oligodendroglial nuclei are dark, and there is a little dark staining of their cytoplasm. Reactive astrocytes are often stained dark brown. Artefacts, in the form of granular black deposits, are apt to occur, especially in grey matter, if the tissue has been kept for more than a month in formalin.

Blocks are taken, in most cases, from one or two levels of the corpus callosum with the adjoining white matter, and from the upper brain-stem. The choice of these sites is to some extent arbitrary, but was originally based on the familiar observation that after a head injury these structures commonly contain small but visible foci of softening, without haemorrhage. It was found that when such foci are present, they are almost always accompanied by a number of smaller lesions, of the type to be described below.

\section{RESULTS}

The lesions revealed by the Weil-Davenport stain consist in tight clusters of active microglial cells. These clusters have a diameter of anything from about $50 \mu$ upwards; the larger ones, a millimetre or more in diameter, correspond to the small softenings seen by naked eye. The smaller ones stand out clearly under the lowest scanning power of the microscope (Fig. 1). It is this fact that gives 


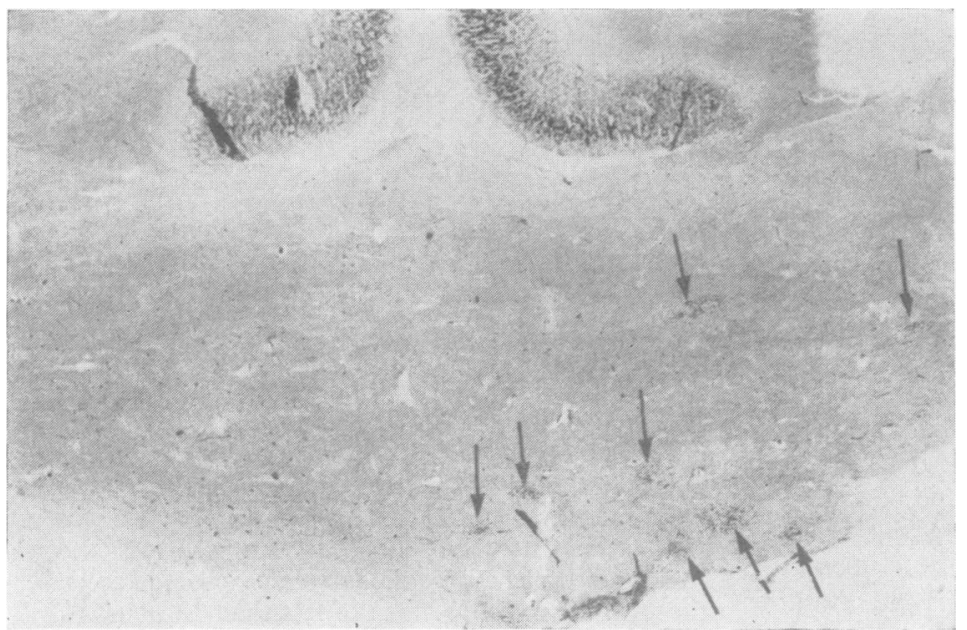

FIG. 1. Corpus callosum, two weeks after a minor head injury. The arrows indicate the positions of microglial clusters. Dark staining in the cingulate cortex is an artefact. WeilDavenport, $\times 6$.

the method its special usefulness. Having found that the lesions are present, one can turn to conventional cell stains, and see that there are local tiny aggregations of microglial nuclei; but this requires higher magnification, and it would be almost impossible to enumerate the lesions in conventionally stained sections.

Lesions of this type were found, in very variable numbers, in approximately three-quarters of the cases examined in this way. Since it was, on the whole, the less severely damaged brains that were selected for examination, and since only one or two blocks were examined in most of the cases, one can safely infer that such lesions occur, in some part of the brain, in more than three-quarters of head injuries. It can also be stated with fair confidence that the lesions are due to trauma. In this department, the Weil-Davenport stain is used a great deal, in a variety of conditions; and I have never observed lesions of this type in cases with no history of trauma.

The shortest period of survival from the time of injury at which I have been able to detect the lesions is 15 hours. At this stage, the microglial cells are still small, and have not developed long pseudopodia (Fig. 2). Between 24 and 48 hours, the reacting microglial cells become larger and more numerous (Fig. 3), and it is possible to observe them, under low magnification, in the form of focal clusters. Some of these are associated with petechial haemorrhages, others not. By the end of this period, axonal retraction balls may be seen in the neighbourhood of the clusters. Myelin stains already show microscopic foci of pallor, but there is little or no astrocytic reaction.

During the first two weeks, the cells composing the clusters enlarge, and form elaborate pseudopodia
(Figs. 4, 7, 8, and 10). They grow plumper in the neighbourhood of a petechial haemorrhage, but do not develop into rounded phagocytes except in the presence of tissue necrosis. At three weeks, reactive astrocytes are seen in the larger and more diffuse $\vec{A}$ lesions, but not in relation to small isolated ones: By the sixth week, swollen-bodied astrocytes may b $\mathscr{C}$ seen around the larger foci, and sudanophil lipide 응 giving a positive Marchi reaction, are seen in frozen sections (Fig. 5). By this time, too, there is often

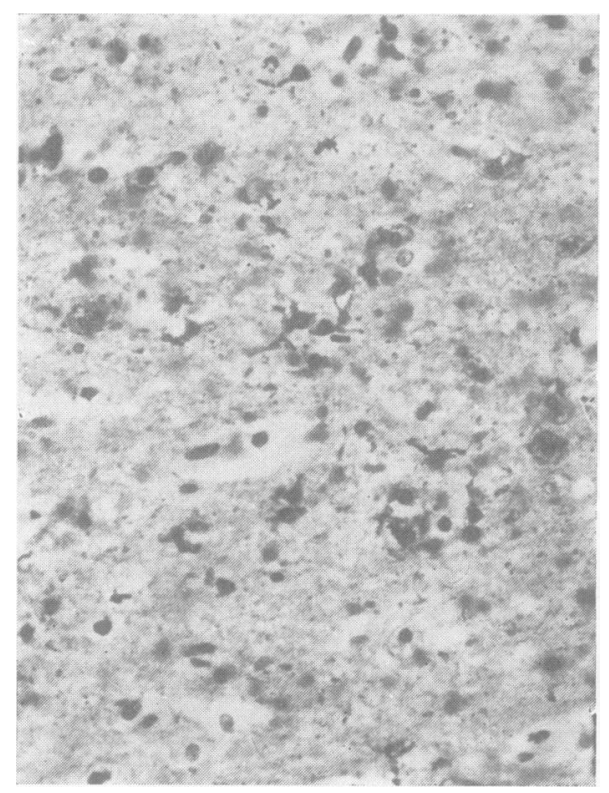

FIG. 2. Corpus callosum, 15 hours after severe head injury. The reacting cells are few and small, with short $N$ processes. Weil-Davenport, $\times 280$. 


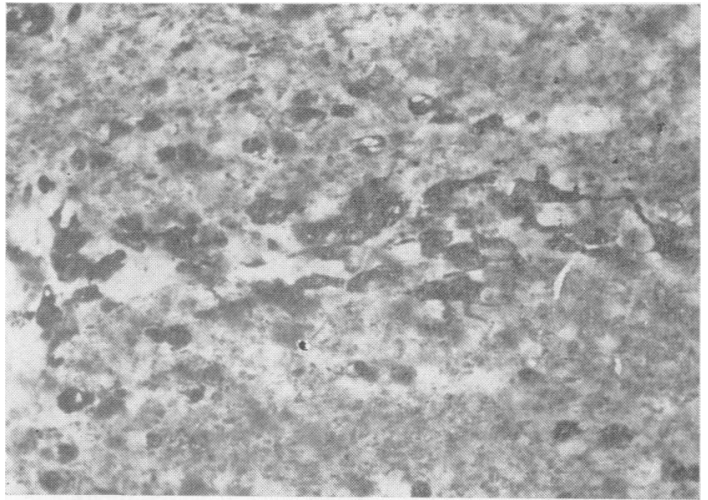

FIG. 3. Corpus callosum, two days after injury. There is a cluster of reactive microglial cells, with short processes. Weil-Davenport, $\times 280$.

diffuse proliferation of microglia, perhaps in response to secondary axonal degeneration. In cases with severe widespread lesions, surviving for months or even years (such as those described by Strich, 1956, 1961), this reaction tends to obscure the individual lesions when they are close together; but isolated microglial clusters, looking very like those seen at four to six weeks, can still be observed.

Little can be said about the distribution of the lesions, owing to the small number of blocks examined. In the corpus callosum, the lesions tend to be concentrated to one or other side of the midline, and are sometimes seen in the adjoining centrum ovale and caudate nucleus. In the upper brain-stem they are more frequent in the tegmentum and brachia conjunctiva than in the cerebral peduncles or basis pontis. They are also seen on the lateral and ventral surfaces of the pons. Lesions are frequently seen immediately alongside a small vessel. They are very infrequent in the cerebral cortex.

Most of the brains examined were from clinically severe cases, dying from the effects of cerebral injury, often combined with cerebral anoxia from chest troubles. It is worth noting, however, that in five of the cases in which microglial clusters were observed the cerebral injury was clinically trivial, consisting of 'concussion', lasting only a few minutes. The cause of death in these was usually fat embolism or pneumonia. A brief account of one such case follows.

CASE J.M. A man of 66 was admitted to hospital with multiple rib fractures, having been knocked down by a motor scooter. He had been stunned, and had a retrograde amnesia of $10-15$ minutes and a post-traumatic

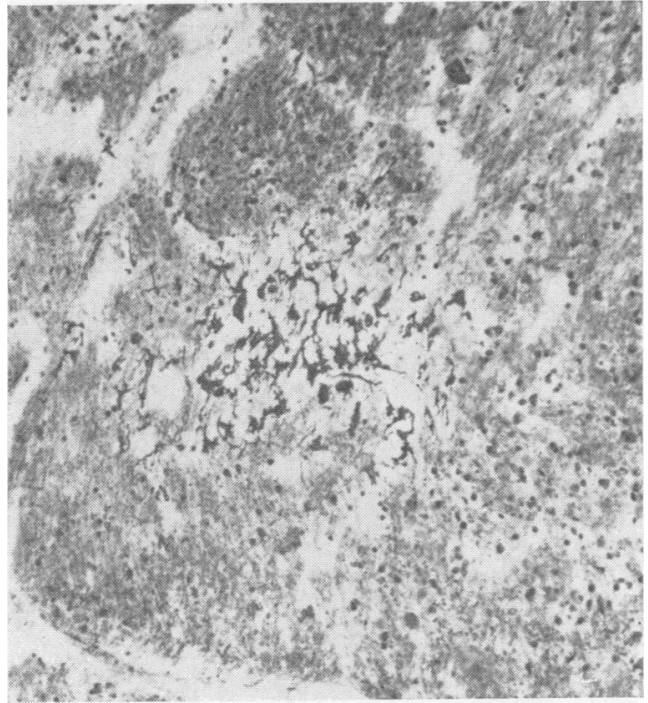

FIG. 4. Lateral part of pons, 10 days after injury. The reactive cells have well-developed pseudopodia. WeilDavenport, $\times 110$.

amnesia of about 20 minutes. There was a parietal bruise, but no skull fracture, and no neurological signs. He was a bronchitic, and died of chest complications 13 days after the injury. At necropsy there was bronchopneumonia, with small abscesses. The brain looked entirely normal except for a tiny softening in the lateral sulcus on one side of the midbrain. There was no vascular disease, and no sign of brain swelling. Histologically, there was some myelin destruction and numerous axonal retraction bulbs in the midbrain lesion. Nine blocks, from various parts of the brain, were stained for microglia. In every block, at least one microglial cluster was found (Figs. 6 to 8).

For comparison, the findings in a case of more severe head injury are as follows.

CASE L.T. A 19-year-old girl drove her car into a telegraph post, and was deeply unconscious from then on, responding to painful stimulation with 'decerebrate' movements. Bruises were seen in the centre of the forehead and over the right temple. There were epileptiform attacks, but no respiratory trouble. No surgery was contemplated. On the fifth day, the pupils became dilated and fixed, and breathing ceased. At necropsy, there were multiple crack fractures at the base of the skull, but no dural tears. The brain was swollen, with some superficial contusions (presumably a contre-coup injury) on the left side, but no gross haemorrhage or laceration. The left uncus was herniated and bruised, and there was cortical infarction in the territory of the anterior temporal branch of the left posterior cerebral artery. On slicing, areas of petechial haemorrhage were seen in the left temporal white matter, on the left side 


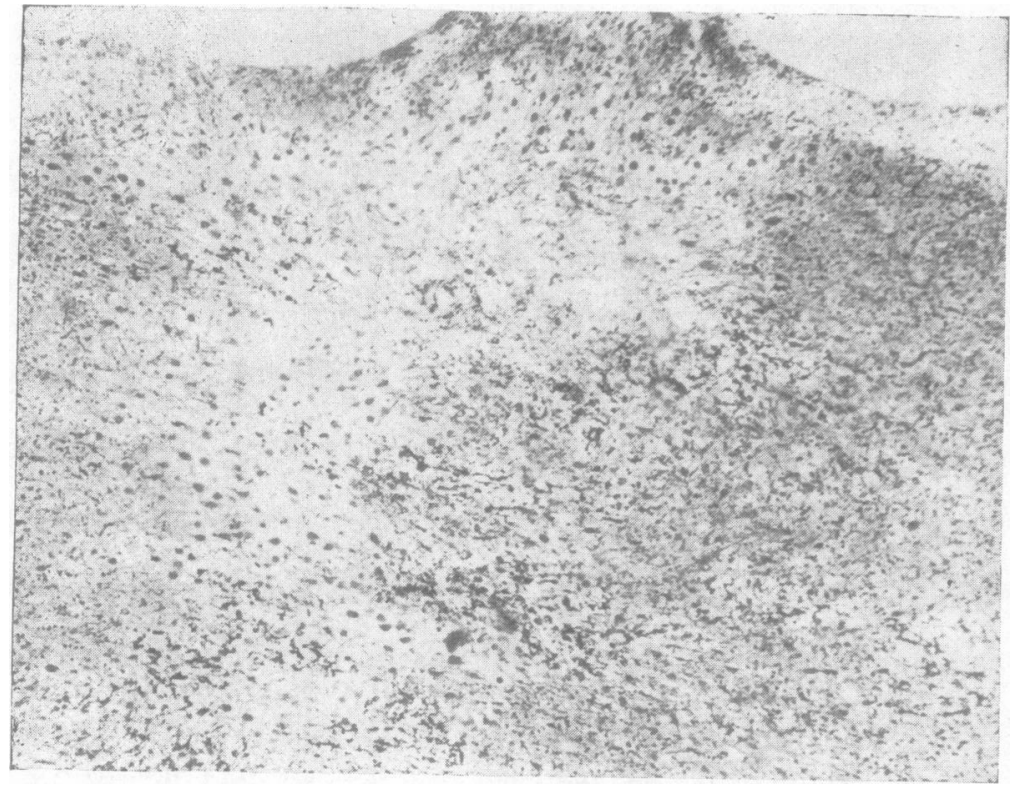

FIG. 5a.

FIG. $5 b$.

FIG. 5. Corpus callosum, six weeks after severe head injury. In (a), the clusters can still be distinguished, but there is diffuse microglial proliferation, and there are numerous swollen astrocytes, staining less intensely. (b) Shows discrete areas of myelin breakdown, of varying size. (a) Weil-Davenport, (b) Marchi, both $\times 45$.

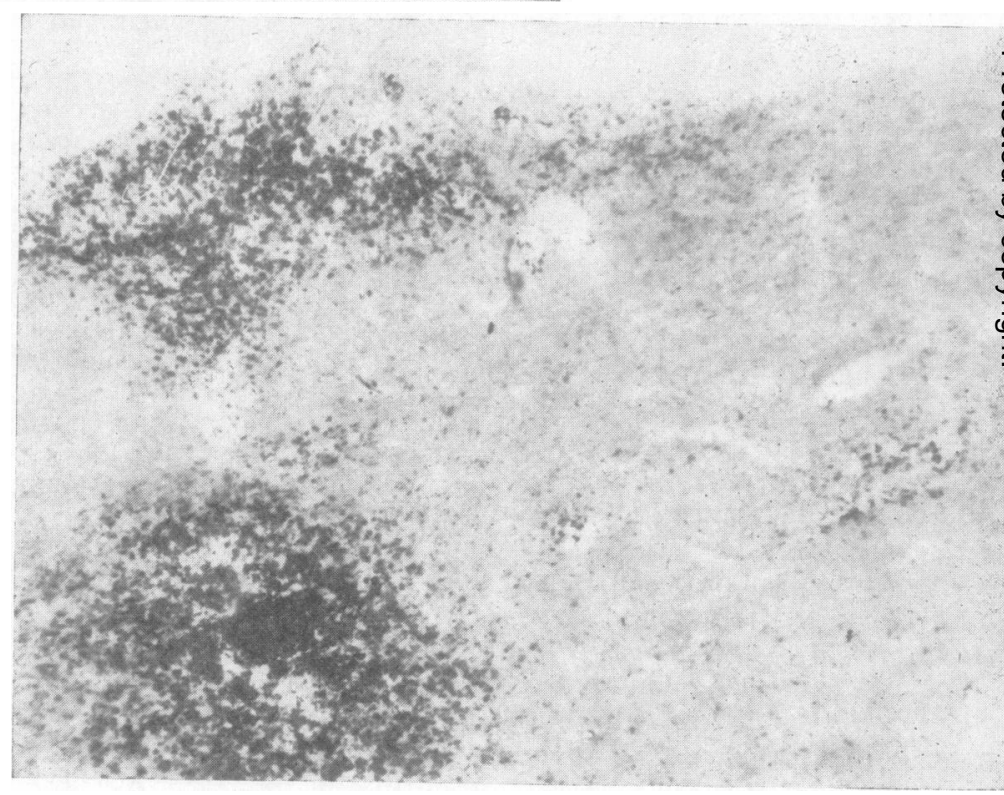

of the corpus callosum, and in the grey matter around the aqueduct. The distribution of the lesions seen in sections from nine blocks is shown in Fig. 9. In the visibly haemorrhagic areas, the clusters are very numerous, and almost confluent: elsewhere, they are isolated and small (Fig. 10). It is interesting that a state clinically labelled 'decerebration' should go with such apparently trivial damage to the brain-stem. One may suspect that the lesions in the area of the dorsal tegmental nucleus, shown in Fig. 9c and d, are important in this context.

\section{DISCUSSION}

There are probably at least four ways in which excessive accelerations may produce scattered microscopic lesions in the brain. First, there is the effect of impact, either direct or shearing, on a hard structure. If this is severe, it will produce a visible contusion. The microscopic lesions often seen on the surface of the upper brain-stem may legitimately be regarded as tiny contusions, due 
perhaps to sudden pressure on the free edge of the tentorium, or on a posterior cerebral artery. Secondly, small vessels in an area of brain undergoing sudden distortion may be stretched and torn, giving rise to haemorrhages of all sizes, down to the tiny capillary haemorrhages which are very frequent in this material. Other theoretical explanations of these haemorrhages have been put forward, and are discussed by Strich (1956). Thirdly, groups of nerve fibres may be similarly stretched and torn, without haemorrhage. The small naked-eye lesions commonly seen in the corpus callosum and brachium conjunctivum are probably of this nature. ${ }^{1}$

${ }^{1}$ The suggestion has been made (Rowbotham, 1949) that the corpus callosum is commonly damaged by impact with the free edge of the falx cerebri. This is implausible, for various reasons. Firstly, the free edge lies about a centimetre away from the corpus callosum, except posteriorly, where it approaches the splenium. It could hardly impinge on the corpus callosum without causing visible damage to an area of cingulate cortex. I have only once seen a brain in which this appears to have happened. Secondly, the lesions in the corpus callosum are usually to one side of the midline, and are more frequent in the lower than in the upper part, as in Fig. 6. Rowbotham's theory is discussed, and rejected, by Lindenberg, Fisher, Durlacher, Lovitt, and Freytag (1955).
Whenever there are naked-eye lesions in the corpus callosum and brachium conjunctivum, microscopic lesions are also present, and microscopic lesions are very commonly present in the absence of macroscopic ones.

The fourth mechanism is a tearing of nerve fibres crossing a blood vessel, which is not itself torn. This is most likely to occur near the surface, where shearing strains are most intense. Its occurrence is suggested by the frequent finding of microglial clusters immediately alongside a blood vessel. Figure 11 shows an example in which a small vessel near the surface of the pons has remained in its displaced position, with kinked nerve fibres on one side, and retraction bulbs and reactive microglial cells on the other.

There can be little doubt that the lesions described here are of the same nature as those described by Strich in 1956 and 1961. Strich examined the brains of 20 patients surviving, in a state of profound dementia, for many months following a head injury. At necropsy, the brains were atrophied, but a.

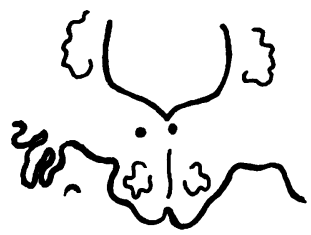

d.

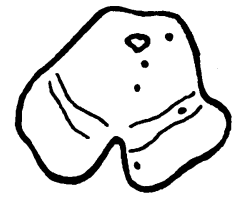

g.

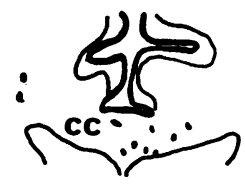

h.

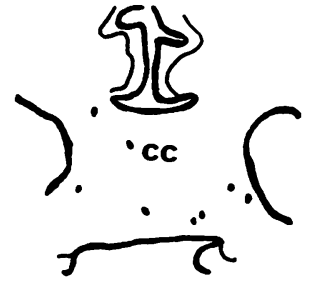

c.

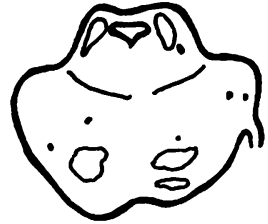

f.

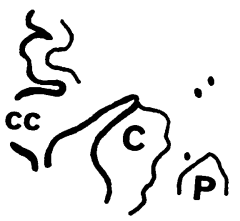

i.

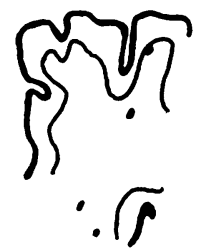

FIG. 6. Case J.M.: 'concussion', with 13 days' survival. Camera lucida tracings from nine blocks. The dots show the distribution of microglial clusters. (a) Upper medulla, (b) lower pons, (c) upper pons, (d) midbrain, (e) left thalamus and insula, (f) right corpus striatum, (g) middle of corpus callosum, (h) splenium of corpus callosum, (i) right parietal parasagittal area. Abbreviations: $\mathrm{C}$, caudate nucleus; cc, corpus callosum; $\mathrm{P}$, putamen; $\mathrm{T}$, thalamus. 

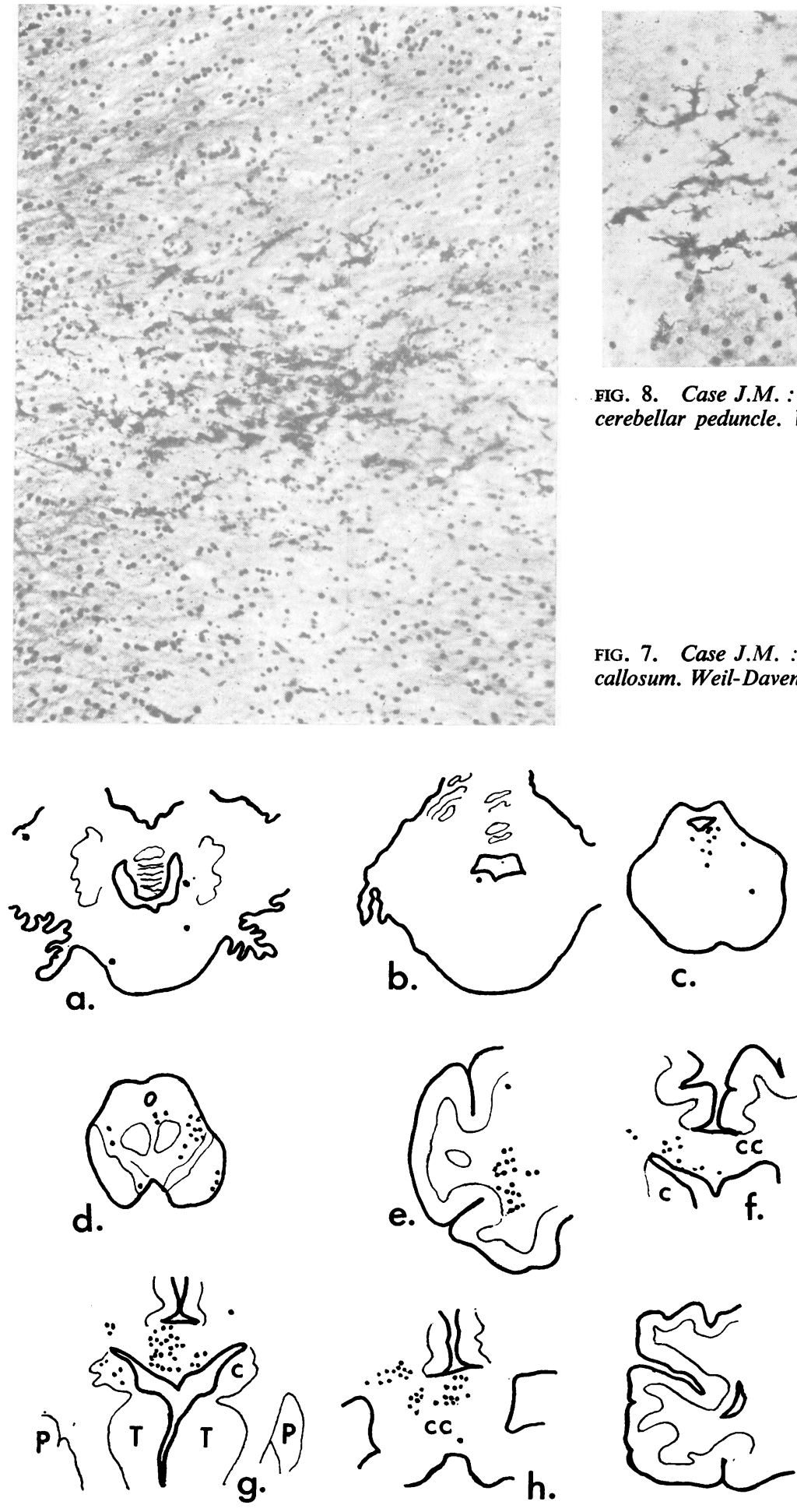

FIG. 7. Case J.M. : 13 days' sur.
callosum. Weil-Davenport, $\times 100$.

. FIG. 8. Case J.M. : 13 days' survival. Lesion in middle cerebellar peduncle. Weil-Davenport, $\times 186$.

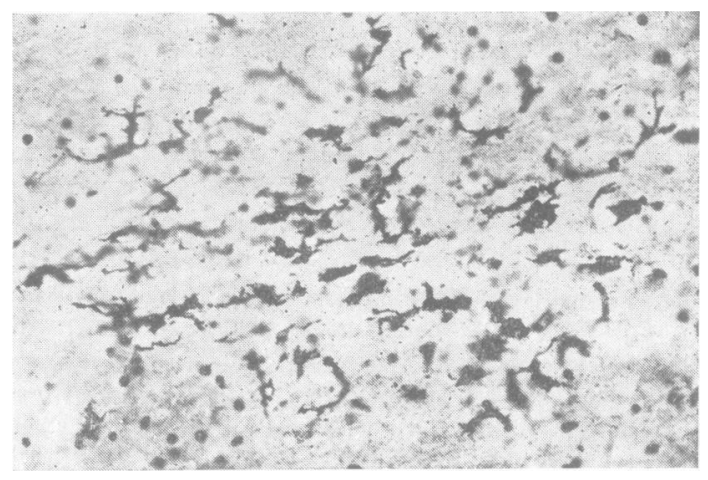

FIG. 9. Case L.T.: five days' survival, in coma. Camera lucida tracings, showing distribution of microglial clusters. (a) Lower pons, (b) mid-pons, (c) upper pons, (d) midbrain, (e) left temporal lobe, anterior part, (f) corpus callosum, anterior part, (g) basal nuclei and corpus callosum, (h) splenium of corpus callosum, (i) right calcarine area. Abbreviations as in Figure 6.

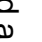




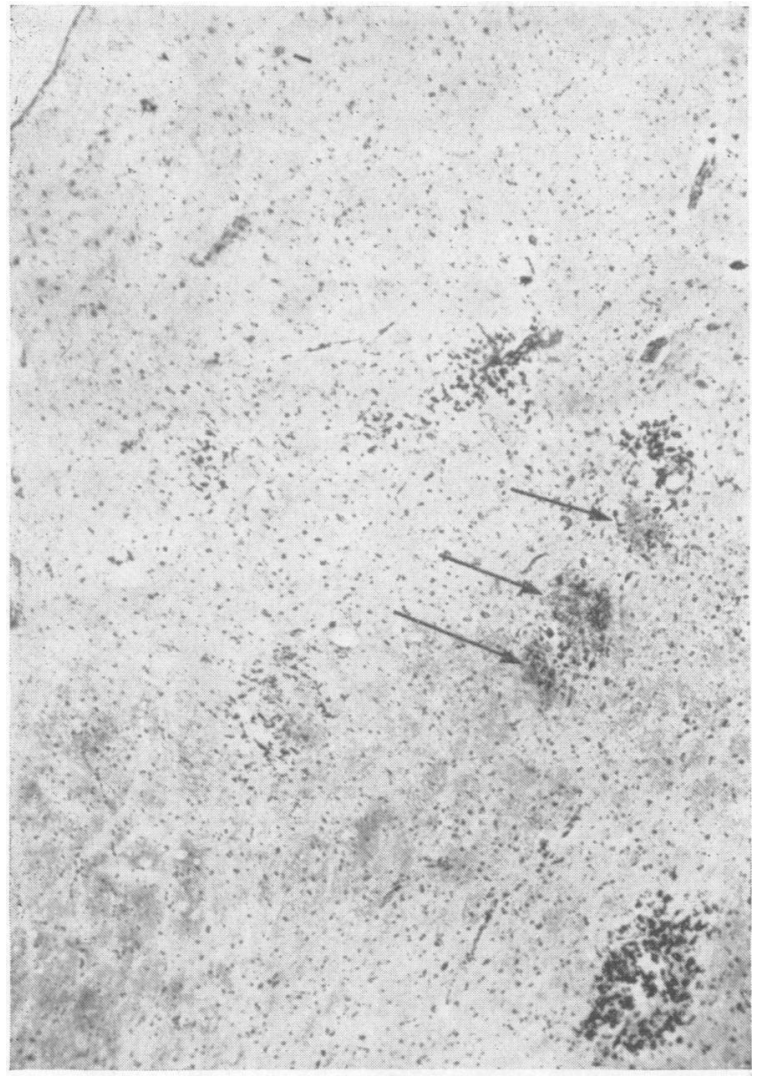

FIG. 10. Case L.T. : five days' survival. Clusters in upper pons, near floor of fourth ventricle (top left corner). Petechial haemorrhages are indicated by arrows. Weil-Davenport, $\times 45$.
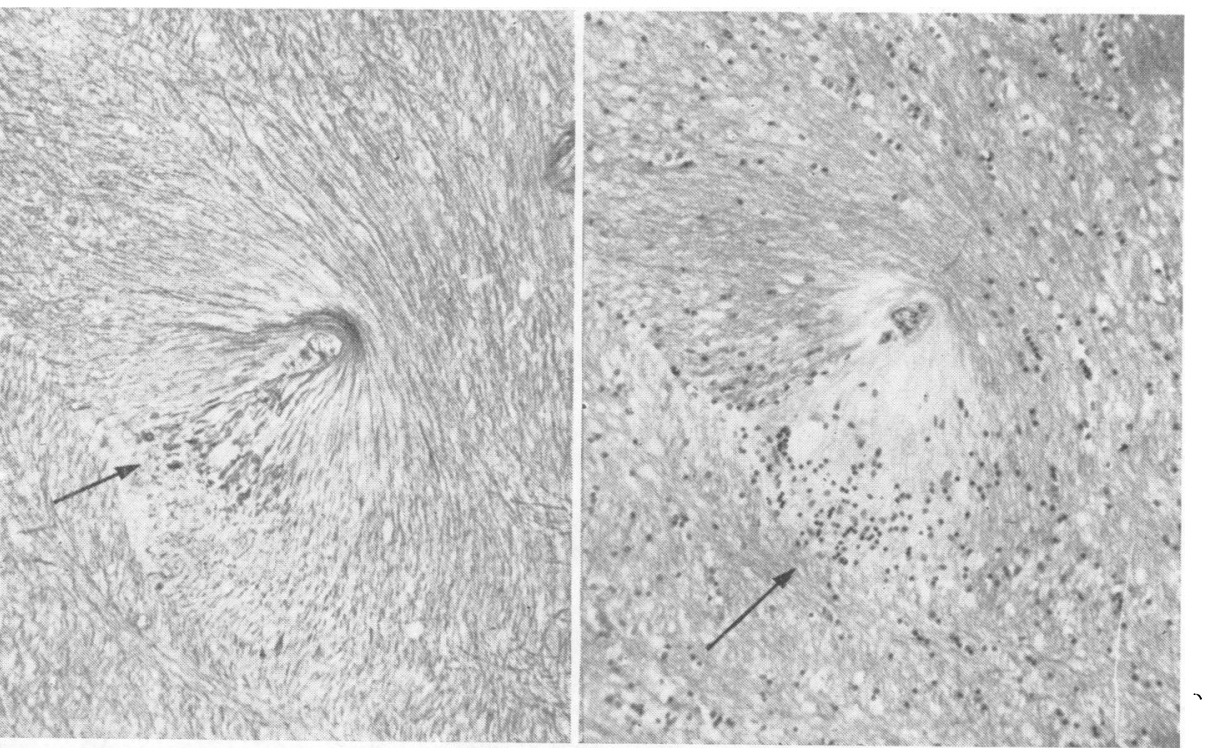

(b)

(a)

FIG. 11. Lateral area of pons, in case with two days' survival. A small vessel has been displaced, with stretching and tearing of nerve fibres. (a) Holmes silver impregnation, showing axonal retraction bulbs (arrow); (b) Haematoxylin and van Gieson, showing cellular reaction (arrow). Both $\times 110$. 
showed no gross lacerations or haemorrhages. Microscopically, Strich demonstrated (mainly by the use of the Marchi technique) numerous tiny lesions in the brain-stem and in the cerebral white matter and basal ganglia. In some preparations, she noted the presence of scattered 'glial stars' in routine cell stains (Strich, 1961, Fig. 7). Comparing her material with mine, I have found lesions such as she describes in cases with survival times of six weeks or more. When Marchi and Weil-Davenport preparations are made from the same block, the identity of the lesions, as seen in the two stains, is obvious. At low magnifications, they are more conspicuous in the Weil-Davenport stain than in the Marchi.

The demonstration of lesions, other than petechial haemorrhages, in cases which were clinically labelled 'concussion' is of some interest. This is not the place to discuss the meaning of the term 'concussion' or its pathological basis. The point to be stressed in regard to these cases of 'concussion' is that permanent damage, in the form of microscopic destructive foci, can be inflicted on the brain by what are regarded as trivial head injuries. If such injuries are repeated (as they may be, for instance, in an unsuccessful boxer), one would anticipate that a progressive, cumulative loss of tissue, and of nervous function, would occur.

It is worth noting that some of the lesions shown in Figs. 6 and 9 correspond to points where sensory evoked potentials were temporarily abolished after concussive blows to the head in experimental monkeys (Foltz and Schmidt, 1956). These authors proposed the theory that the loss of consciousness is primarily due to a transient depression of activity in the ascending reticular formation. No lesions were observed in the brain-stems of these animals, which were sacrificed immediately after the experiment. It would be interesting to look for microglial reactions, say, three days after such experiments.

The method described in this paper could, I think, be a useful tool in investigating the mechanics of acceleration injuries. For instance, it would be of interest to study the directions in which the torn nerve fibres are running in various parts of a single brain; to correlate the distribution of lesions with the theoretical axis of acceleration; and to attempt a correlation between the numbers, and sites, of the lesions and the recorded disturbances of nervous function. In this department, the method has already given useful information in cases with profound neurological disturbances, but with very little naked-eye damage to the brain.

\section{SUMMARY}

Following a head injury, diffuse microscopic lesions can be seen in a high proportion of human brains. These consist of $(a)$ capillary haemorrhages and (b) severing of nerve fibres without haemorrhage. There is a local microglial reactior, which can be demonstrated with survival times of less than 24 hours after the injury. This reaction is readily seen with the lowest power of the microscope, in frozen sections stained with a simple silver technique. The lesions are still visible many months after injury, and appear to be identical with those described by Strich (1956) in cases with long survival. They are believed to be mechanical in origin, and can be attributed to (1) surface shearing and contusion; (2) stretching and tearing of small blood vessels; (3) stretching and tearing of groups of nerve fibres; (4) tearing of nerve fibres by a crossing i vessel. They are seen, not only after severe traumad but also in cases of 'concussion'. Detailed studies o their sites and distribution could throw light on the mechanics of acceleration injuries of the brain.

The author is grateful to Mr. R. A. Beesley for the histological preparations in this series.

\section{REFERENCES}

Foltz, E. L., and Schmidt, R. P. (1956). The rôle of the reticular formation in the coma of head injury. J. Neurosurg., 13, 145-154.

Lindenberg, R., Fisher, R. S., Durlacher, S. H., Lovitt, W. V., and Freytag, E. (1955). Lesions of the corpus callosum following blunt injury to the head. Amer. J. Path., 31, 297-318.

Rowbotham, G. F. (1949). Acute Injuries of the Head, 3rd ed. (Chapter 1). Livingstone, Edinburgh.

Strich, S. J. (1956). Diffuse degeneration of the cerebral white matter in severe dementia following head injury. J. Neurol. Neurosurg Psychiat., 19, 163-185.

(1956a). The Pathological Changes in the Central Nervous System of Patients with Profound Traumatic Dementia. D.M. Thesis, Oxford.

(1961). Shearing of nerve fibres as a cause of brain damage due to head injury. Lancet, 2, 443-448.

Weil, A., and Davenport, H. A. (1933). Staining of oligodendroglia and microglia in celloidin sections. Arch. Neurol. Psychiat. (Chic.), 30, 175-178. 